\title{
Doença de Crohn e Farmacobezoar Intestinal: Relato de Caso
}

\author{
Crohn's Disease and Bowel Pharmacobezoar: Case Report
}

\author{
ADRIANA SANTOS NEVES TRECE ${ }^{1}$; LUCIANAPAES PEIXOTO NETTO ${ }^{1}$; RAFAELLIMATRECE ${ }^{1}$; \\ FERNANDO PINTO BRAVO $^{1}$; PAULO CESAR CASTRO JÚNIOR $^{1}$; FRANCISCO LOPES PAULO $^{2}$
}

\begin{abstract}
${ }^{1}$ Médico da Disciplina de Coloproctologia do Hospital Universitário Pedro Ernesto - Faculdade de Ciências Médicas Universidade do Estado do Rio de Janeiro; ${ }^{2}$ Chefe da Disciplina de Coloproctologia do Hospital Universitário Pedro Ernesto - Faculdade de Ciências Médicas - Universidade do Estado do Rio de Janeiro.
\end{abstract}

\begin{abstract}
TRECE ASN; PEIXOTO NETTO LP; TRECE RL; BRAVO FP; CASTRO JÚNIOR PC; PAULO FL. Doença de Crohn e Farmacobezoar Intestinal: Relato de Caso. Rev bras Coloproct, 2010;30(2): 215-220.

RESUMO: Bezoares são concreções de materiais diversos, parcialmente ou não digeridos, que podem ser encontrados em qualquer porção do tubo digestivo. Raramente são compostos por fármacos, nestes casos denominados farmacobezoares. Este artigo visa relatar o caso de um paciente com Doença de Crohn complicada por fístula entero-cutânea e áreas de estenoses no íleo terminal apresentando bezoar de mesalazina. Foi enfatizado o caráter multifatorial da gênese dos farmacobezoares, assim como a variada gama de apresentações clínicas e exames diagnósticos úteis no esclarecimento dos casos. Devemos ainda salientar a necessidade de individualização do tratamento para melhor eficácia do mesmo.
\end{abstract}

Descritores: Bezoar; Farmacobezoar; Doença de Crohn; Mesalazina; Estenose de delgado.

\section{INTRODUÇÃO}

O termo bezoar deriva de bazahr ou badzehr, antídoto em árabe. Pois, na medicina, até o século XVI, aglomerados de fibras vegetais encontradas no trato digestivo proximal de herbívoros eram utilizadas como tratamento para diversas patologias. Por analogia, qualquer outro corpo parcialmente digerido formando massa no tubo digestivo passou a ser designado por "bezoar"(1).

Diversos são os tipos de bezoares. Dentre eles, os mais comumente descritos são: fitobezoar, tricobezoar, farmacobezoar, entre outros ${ }^{(2)}$.

Podem se formar em qualquer porção do trato gastrointestinal (TGI) e comumente estão associados a fatores predisponentes anatômicos, funcionais ou outras patologias concomitantes ${ }^{(2,3)}$. As propriedades farmacológicas das diferentes drogas também estão intimamente ligadas à fisiopatologia dos farmacobezoares ${ }^{(3)}$.
As manifestações clínicas são variadas, desde casos assintomáticos (maior parte) até oclusão intestinal. Quando se trata de farmacobezoares, sintomas de overdose da medicação podem estar presentes ${ }^{(4)}$.

Diferentes exames são úteis na investigação diagnóstica destes pacientes, incluindo-se os radiológicos simples ou contrastados e a avaliação endoscópica ${ }^{(3,4,5,6,7)}$.

Não existe padrão-ouro para o tratamento dos farmacobezoares. Esse deve ser individualizado para cada caso e diversas são as opções ${ }^{(2)}$.

Relatar-se-á neste artigo o caso de um paciente portador de Doença de Crohn (DC) complicada por fístula entero-cutânea e estenoses de delgado com formação de farmacobezoar de mesalazina. O mesmo foi tratado cirurgicamente pelos médicos do Serviço de Coloproctologia do Hospital Universitário Pedro Ernesto (HUPE) da Universidade do Estado do Rio de Janeiro (UERJ).

Trabalho realizado no Hospital Universitário Pedro Hernesto - Faculdade de Ciências Médicas - Universidade do Estado do Rio de Janeiro - RJ - Brasil.. 


\section{RELATO DO CASO}

M.J.F.P., 50 anos, sexo masculino, branco, natural do Rio de Janeiro. Diagnóstico de DC há 14 anos, após tratamento cirúrgico para fístula entero-vesical, permanecendo 9 anos sem tratamento regular. Encaminhado ao ambulatório de Doenças Inflamatórias Intestinais (DII) do serviço de Gastroenterologia do HUPE sendo iniciada terapia clínica regular. Após um ano de acompanhamento apresentava fístula enterocutânea (Figura 1), cólicas abdominais e náuseas não responsivas à terapêutica clínica com o uso de mesalazina, corticosteróides e infliximabe. Solicitado então parecer à Coloproctologia para avaliação e tratamento cirúrgico.

A videocolonoscopia pré-operatória identificou estenose anelar no cólon ascendente e ceco, com pólipos inflamatórios e ulcerações superficiais, envolvendo a válvula ileocecal. Havia trânsito de secreção entérica pela estenose. O íleo terminal não pode ser avaliado, porém a insuflação de ar nesta região provocava aumento do débito gasoso no óstio cutâneo. A TC e o Clister Opaco pouco acrescentaram ao estudo do caso. $\mathrm{O}$ exame de trânsito de delgado mostrou áreas de estenoses no íleo terminal adjacentes a segmentos com dilatação importante onde se identificavam falhas de enchimento múltiplas, arredondadas, de contornos regulares, homogêneas e uniformes de $1.2 \mathrm{~cm}$ (Figura 2) e trajeto fistuloso para pele a partir desta mesma região. $O$ contraste não progrediu até o ceco.

Submetido a laparotomia exploradora em 13/ 08/07 sendo identificadas 4 áreas de estenose em íleo terminal, a mais distal junto à válvula ileocecal, acometendo e deformando o ceco, com fístula enterocutânea. Presença de regiões com dilatação importante do delgado, anteriores às estenoses, com acúmulo de comprimidos de mesalazina nestes "bolsões" (Figuras 3 e 4). Realizada ileotiflectomia com ressecção da pele em bloco. Enterectomia segmentar (10 cm de íleo) englobando duas das áreas de estenose. Estrituroplastia do segmento estenosado mais proximal (Figuras 5 e 6 ).

O pós-operatório transcorreu sem

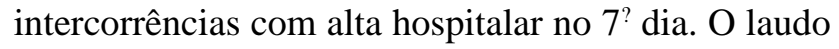
histopatológico revelou: segmento ileocecal de $18 \mathrm{~cm}$ com processo inflamatório crônico, fistulizado, compatível com Doença de Crohn (Figura 7). Segmento ileal de $10.5 \mathrm{~cm}$ com ileíte crônica, porém sem granulomas (Figura 8). Ausência de malignidade nas peças.

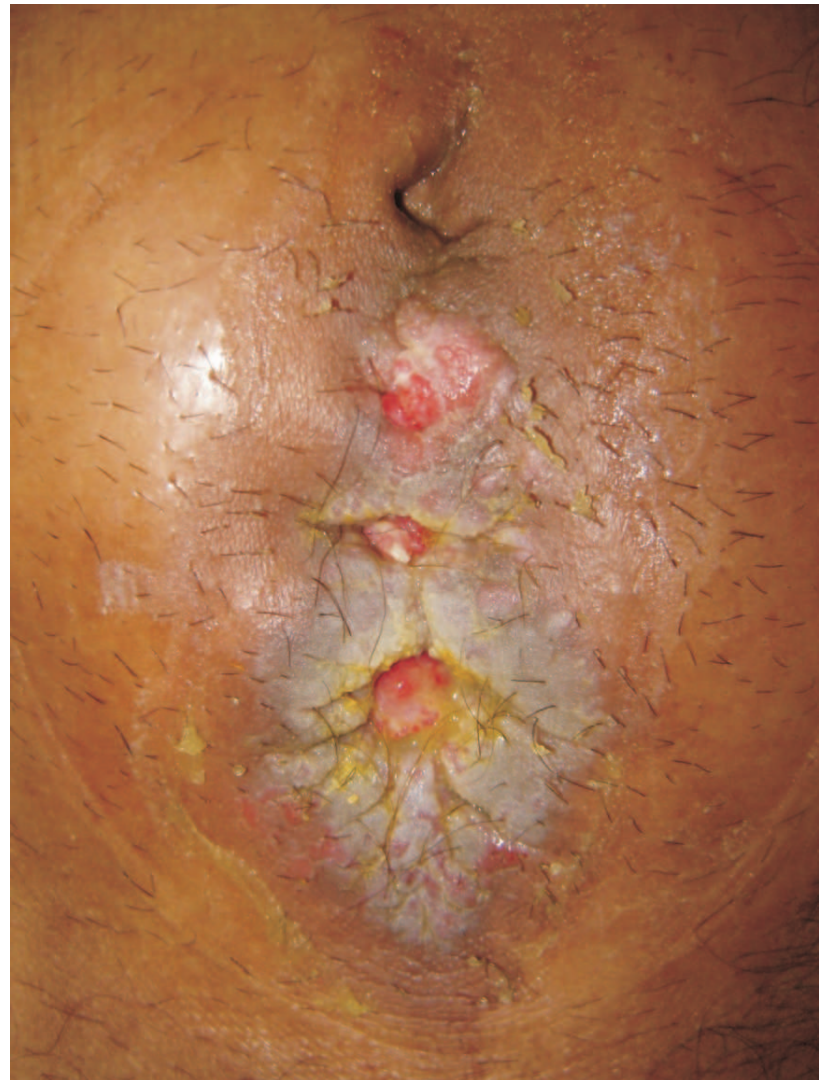

Figura 1 - fístula entero-cutânea.

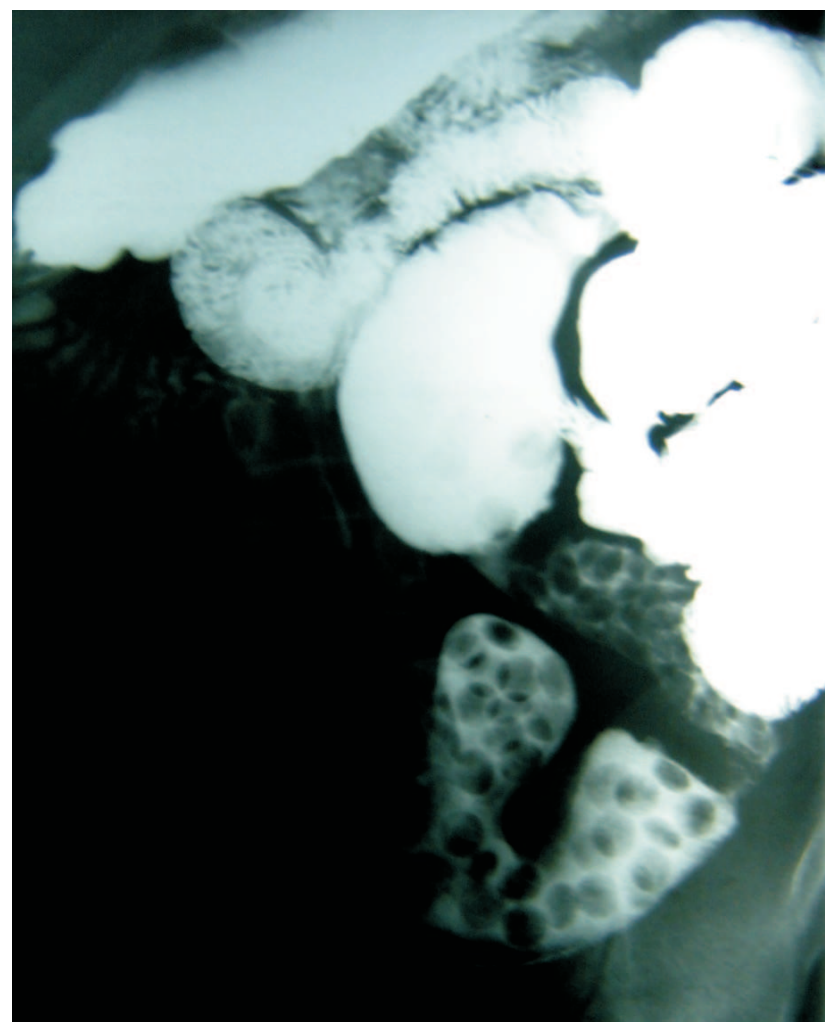

Figura 2 - Eestenoses e falhas de enchimento no trânsito de delgado. 


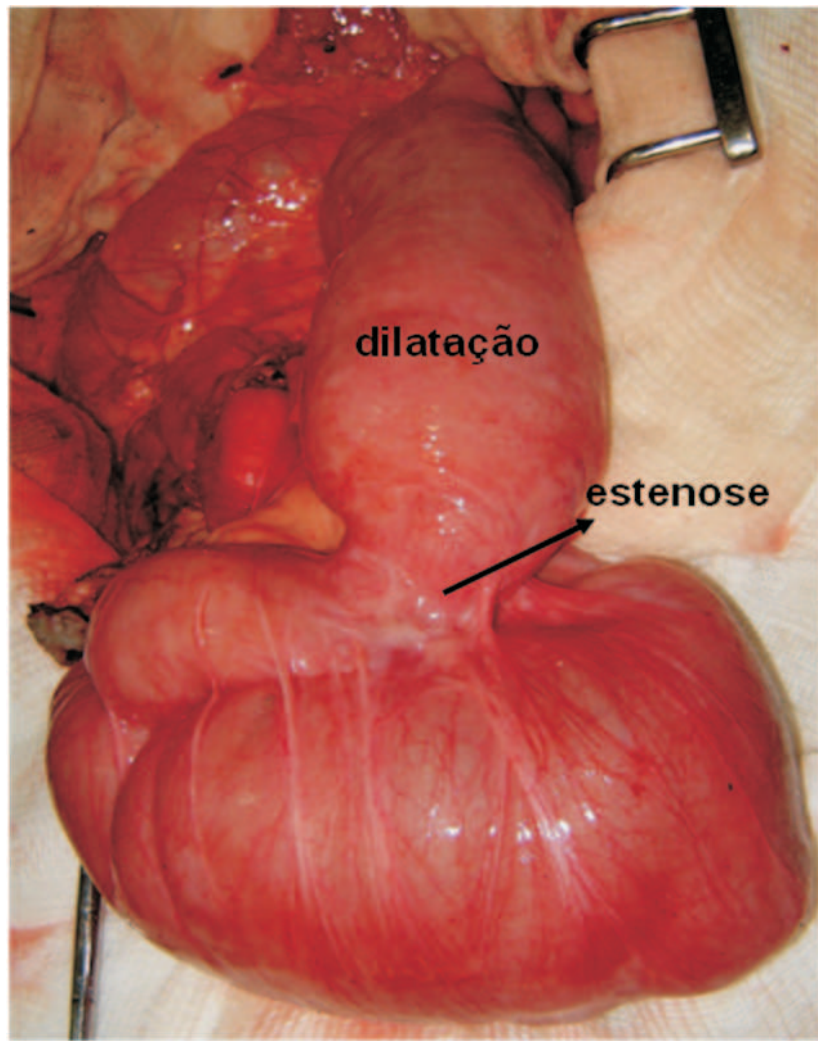

Figura 3 - Dilatações e estenoses do íleo terminal,

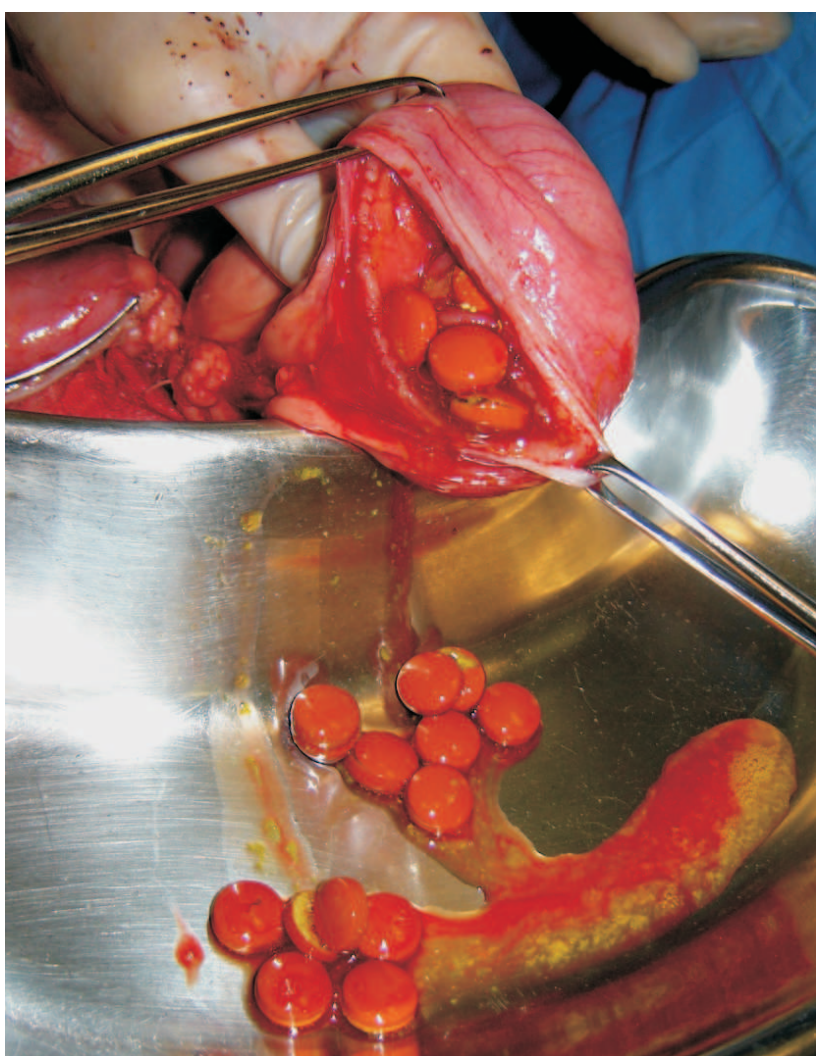

Figura 4 - Farmacobezoar de mesalazina.

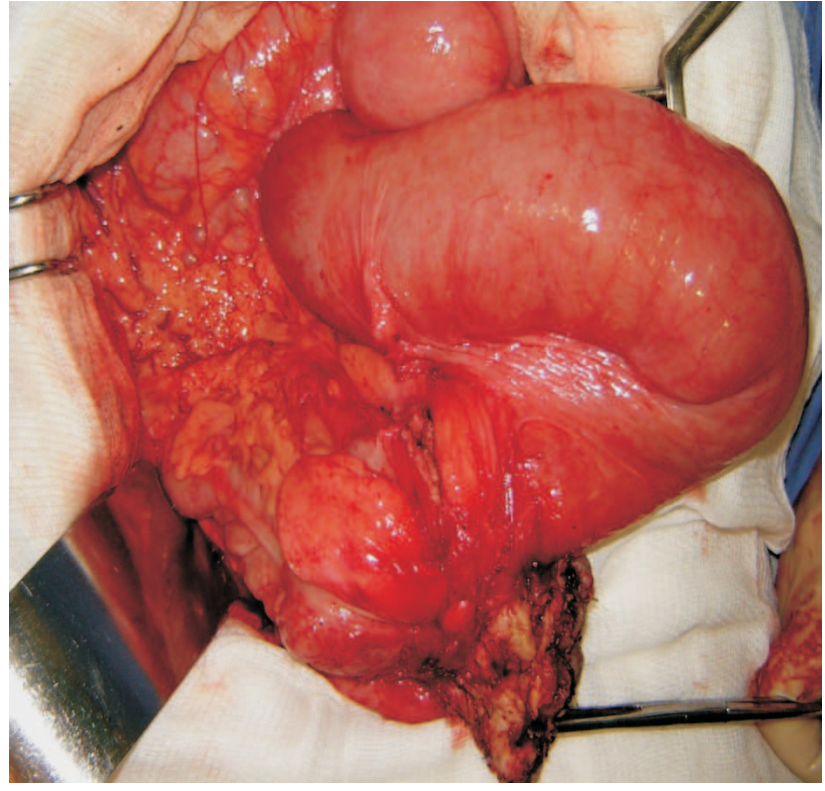

Figura 5 - Ressecção em bloco da fístula entero-cutânea.

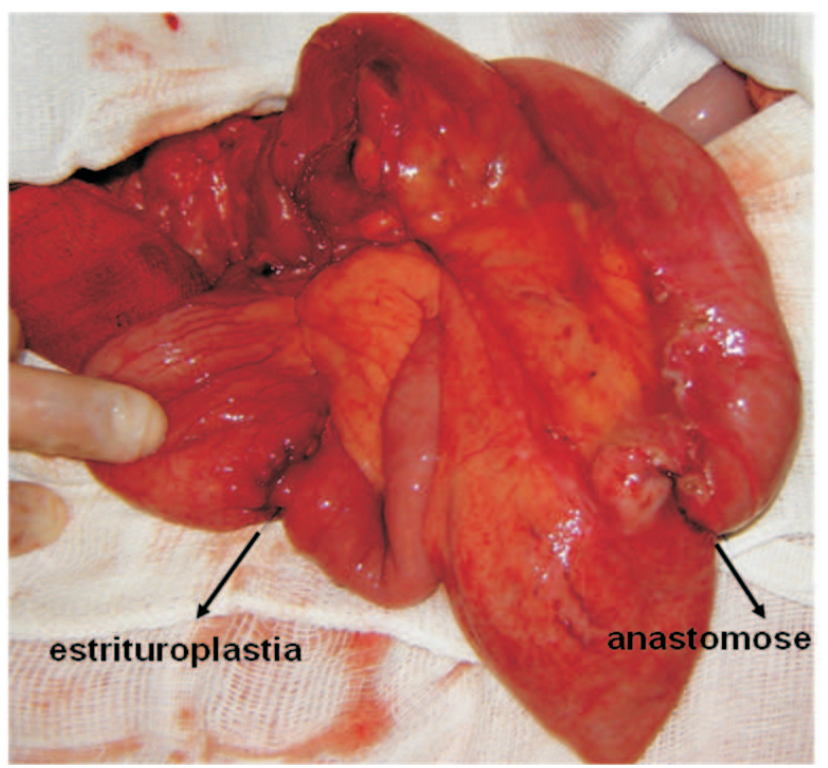

Figura 6-Enterectomia e estrituroplastia.

\section{DISCUSSÃO}

Bezoares são concreções de materiais diversos, parcialmente ou não digeridos, que podem ocorrer em qualquer porção do TGI. Algumas vezes formados por fibras (fitobezoares), cabelo (tricobezoares, frequentemente em pacientes psiquiátricos), medicamentos (farmacobezoares) ou outros componentes (tais como areia e pedras, sobretudo em crianças). 


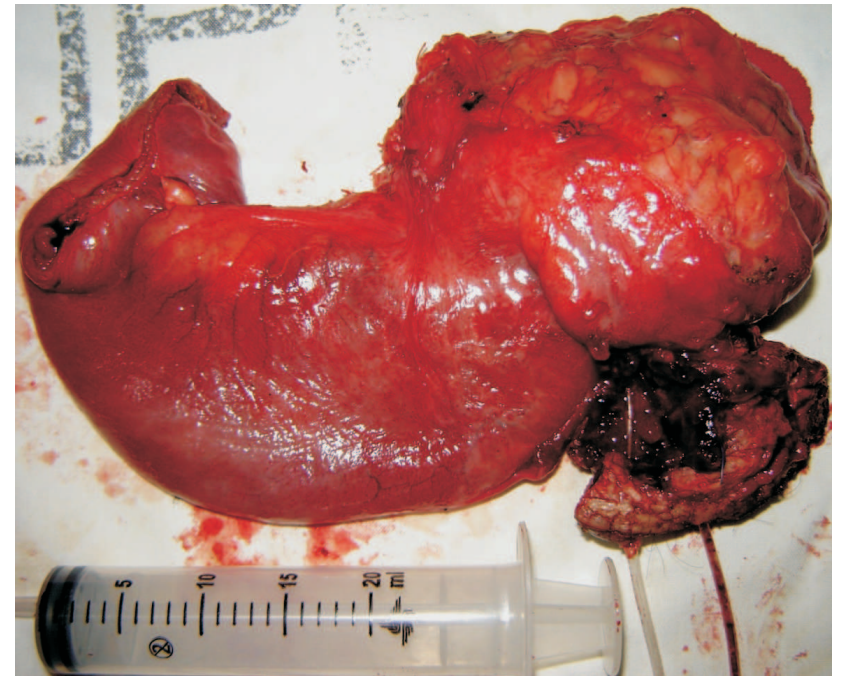

Figura 7-Segmento ileocecal.

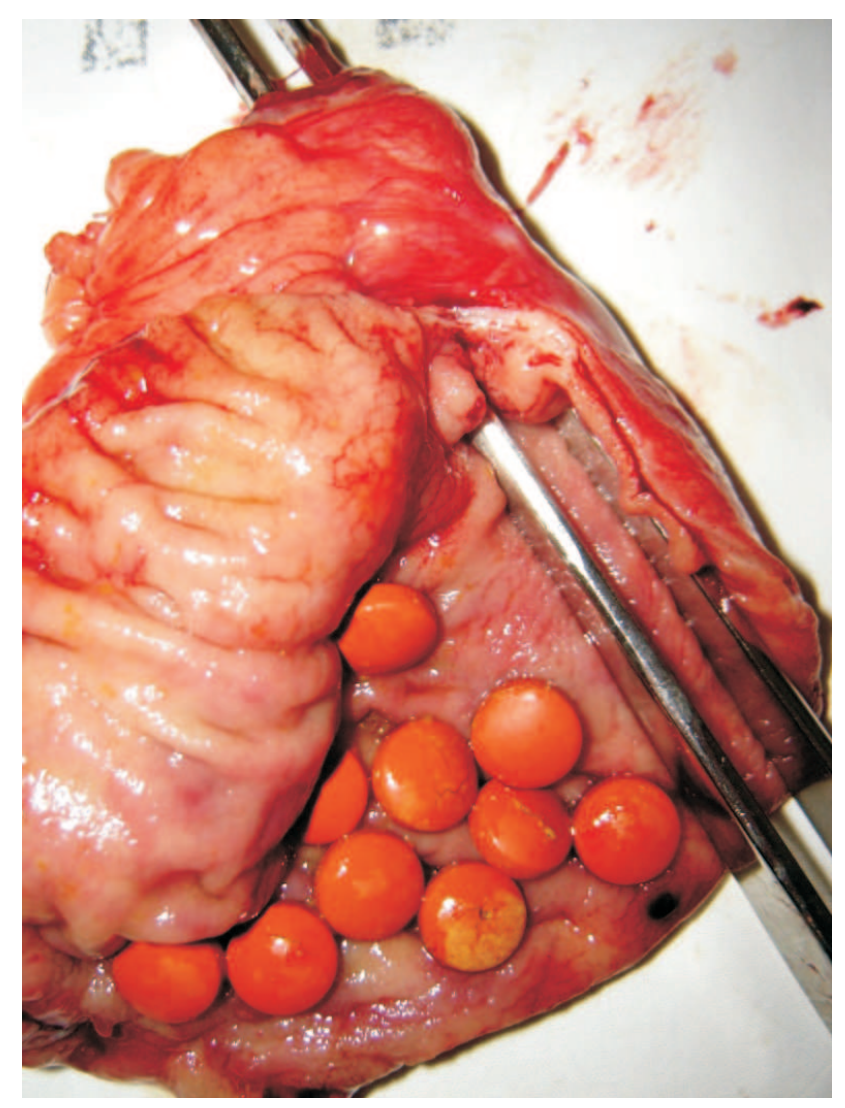

Figura 8-Segmento de delgado com comprimidos de mesalazina.

Os farmacobezoares estão intimamente relacionados às propriedades farmacológicas das medicações e a fatores predisponentes individuais, sejam anatômicos ou funcionais e patológicos ou constitucionais. Os bezoares ocorrem com maior frequência no estômago, esôfago e mais raramente no intestino delgado ou cólon ${ }^{(2)}$.

Dentre os fatores de risco inerentes aos remédios podemos citar as formulações revestidas, de absorção tardia, seja entérica ${ }^{(8)}$ ou mais distalmente no tubo digestivo. Há também aquelas de liberação contínua, com cápsula constituída de membrana semipermeável, que não alteram seu volume durante o processo digestivo ${ }^{(2,3,4)}$. Dentro desse contexto podemos observar os frequentes relatos de farmacobezoares compostos de verapamil ${ }^{(9,10)}$, nifedipina ${ }^{(3,6,11,12)} \mathrm{e}$ teofilina ${ }^{(4,13)}$ de liberação lenta. Pacientes que ingerem diariamente um grande número de pílulas também estão sob maior risco ${ }^{(7,14)}$.

Encontramos ainda na literatura alguns outros medicamentos envolvidos na formação de bezoares, entre eles podemos citar: aspirina revestida ${ }^{(8,15)}$, nelfinavir ${ }^{(14)}$, vitamina $\mathrm{B} 12^{(5)}$, antiulcerosos ${ }^{(2,7)}$ como sucralfato, hidróxido de alumínio e alginato de sódio, carbono ativado $^{(16)}$, nutrição enteral ${ }^{(17)}$ e laxantes formadores de bolo fecal ${ }^{(2)}$ (farmaco-fitobezoares).

Dificilmente observamos a formação de bezoares no tubo digestivo sem que nesse haja alterações anatômicas, funcionais ou existam outras doenças associadas. Logo, podemos citar os seguintes fatores envolvidos: estenoses inflamatórias, tumorais, pós-cirúrgicas, secundárias à doença diverticular do cólon $^{(5,18)}$, úlcera péptica, pâncreas anular, estenose pilórica; distorção anatômica decorrente de Divertículo de Meckel; alterações funcionais da motilidade gastrointestinal por uso de anticolinérgicos, narcóticos, pós-operatórias, associadas à gastrectomia ${ }^{(17)}$, vagotomia, pancreatite, colecistite, neuropatias com gastroparesia (diabética); utilização de medicações antiulcerosas que alteram o $\mathrm{pH}$ do TGI; outras patologias como a colestase, doenças psiquiátricas e a mastigação incompleta ${ }^{(1,2,3,7)}$.

O quadro clínico atribuído aos farmacobezoares é variado e inespecífico. Podem ocorrer cólicas abdominais, náuseas, vômitos ${ }^{(7)} \mathrm{e}$, quando não diagnosticados, até mesmo ulceração, hemorragia, sintomas de overdose $^{(3)}$, oclusão ou suboclusão intestinal ${ }^{(3,17,18,19)} \mathrm{e}$ perfuração ${ }^{(2)}$. Porém, na grande maioria dos casos, trata-se de patologia assintomática ${ }^{(2)}$.

O diagnóstico pode ser sugerido por imagens radiológicas em radiografias simples ${ }^{(5)}$, exames contrastados ${ }^{(3,6)}$, ultrassonografia ${ }^{(7)}$ e até mesmo tomografia computadorizada ${ }^{(6)}(\mathrm{TC})$. Em outros casos, os exames endoscópicos com visualização dire- 
ta da concreção, sua fragmentação e remoção confirmam o diagnóstico e agem como opção terapêutica eficaz ${ }^{(4)}$.

Não existe padrão-ouro para o manejo desta doença. $\mathrm{O}$ tratamento deve ser individualizado para

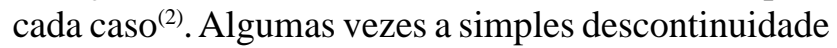
das medicações envolvidas é suficiente ${ }^{(7)}$. Em outras pode ser necessária intervenção com dissolução enzimática $^{(19)}$ (celulase, tripsina, papaína, acetilcisteína, etc), lavagem do TGI, fragmentação e/ou retirada endoscópica ${ }^{(20)}$ e até mesmo procedimentos cirúrgi$\cos ^{(5,6,18)}$

No caso exposto neste artigo, os autores relatam um caso inédito de farmacobezoar por comprimidos de mesalazina. Diferente de outras formulações não protegidas de mesalazina, que têm sua absorção iniciada no trato digestivo superior, o princípio ativo da formulação comercial utilizada pelo paciente só é liberado em $\mathrm{pH}$ maior que 7 , iniciando sua ação apenas no íleo terminal e cólon ${ }^{(21)}$.

A função dos derivados do aminossalicilato nas DII se dá por sua ação tópica. Logo, a eficácia depende da liberação luminal da substância ativa no segmento afetado ${ }^{(22)}$. Drogas liberadas em sítio alvo de TGI têm maior efeito terapêutico e menores efeitos colaterais $^{(23)}$. A mesalazina de liberação intestinal está indicada para a manutenção da forma de íleo-colite na DC. Na indução de remissão de um episódio agudo leve a moderado seu uso ainda é questionável ${ }^{(24)}$.

O caso clínico aqui relatado descreve um paciente com a forma estenosante e fistulizante da DC não responsiva à terapêutica clínica, mesmo com uso de infliximabe. Os sintomas persistentes de cólicas intestinais e náuseas podem ser atribuídos à doença de base, pela presença das estenoses de íleo terminal.

O farmacobezoar pode ter contribuído para a sintomatologia descrita acima. Porém, seu diagnóstico foi um achado incidental durante investigação pré- operatória com exame contrastado (trânsito de delgado) anterior ao tratamento cirúrgico da fístula entero-cutânea. Estas imagens demonstraram falhas de enchimentos múltiplas, homogêneas e regulares, compatíveis com o diagnóstico de farmacobezoar. Esse se encontrava em "bolsões" de delgado dilatado entre as áreas de estenose. Durante a cirurgia para correção das estenoses e fístula o bezoar de mesalazina foi confirmado, com a identificação de vários comprimidos não digeridos à abertura da alça de delgado (Figura 4).

As áreas estreitadas do intestino não permitiram a chegada das pílulas ao seu local de absorção e ação, provocando o acúmulo da medicação e impedindo seu efeito terapêutico tópico (íleo terminal e cólon). O laudo histopatológico confirmou doença ativa na área da fístula e apenas inflamação crônica do delgado envolvido nas zonas de estenose.

\section{CONCLUSÃO}

Com a análise deste relato podemos concluir que uma alteração anatômica pré-existente (estenose do intestino delgado), causada pela doença de base (DC), aliada às propriedades farmacológicas da mesalazina sob a forma comercial utilizada, foram fatores determinantes para a formação do farmacobezoar. $\mathrm{E}$, concomitantemente, impediu-se que princípio ativo atingisse o local de atividade da doença (íleo terminal), onde seria liberado para exercer seus efeitos antiinflamatórios tópicos. A sintomatologia apresentada pelo paciente foi atribuída à DC. O bezoar apresentou-se como um achado incidental durante investigação diagnóstica, porém, em algum grau, pode ter contribuído para o quadro clínico apresentado pelo paciente. Ambas as condições foram resolvidas através do procedimento cirúrgico adotado. Desta forma, reafirmamos o caráter individualizado da clínica e manejo terapêutico nos casos de farmacobezoares.

\footnotetext{
ABSTRACT: Bezoars are masses composed of foreign material, partially digested or not, found in any portion of the gastrointestinal tract. Rarely bezoars are composed by medication tablets, becoming known as Pharmacobezoars. This article reports a patient with the Crohn's disease complicated by enteric fistulae and stenotic areas in the ileum presenting Mesalazine pharmacobezoars. The author emphasizes the multi-factorial origin of pharmacobezoars, the wide range of clinical presentations and the diagnostic tools useful in these cases. The individualized treatment necessary to obtain the satisfactory clinical results is also highlighted.
}

Key words: Bezoar; Pharmacobezoar; Crohn disease; Mesalazine; Small intestine stenosis. 


\section{REFERÊNCIAS}

1. Oliveira GA, Werlang HZ, Bergoli PM, Abikair-Filho J. Plastibezoar no íleo terminal: relato de caso e aspectos de imagem. Rev Imagem 2006; 28 (2): 117-120.

2. Pozo JFM, Martínez MS, Porras IC, Torné GR, Luque AC. Farmacobezoar em um paciente intervenido de estenosis pilórica. Farm Hosp 2004; 28 (1): 59-63.

3. Yeen WC, Willis IH. Retention of extended release nifedipine capsules in a patient with enteric stricture causing recurrent small bowel obstruction. South Med J 2005; 98 (8): 839-42.

4. Cereda JM, Scott J, Quigley EM. Endoscopic removal of pharmacobezoar of slow release theophylline. Br Med J 1986; 293 (6555): 1143.

5. Silbergleit R, Lee DC. Bowel Obstruction and Radiopaque Vitamin B12 "Pseudobezoar”. Am J Emerg Med 1995; 13 (1): 112-3

6. G Khera, R Lord. An unusual case of right iliac fossa mass Pharmacobezoar mimicking inflammatory bowel disease. Colorectal Dis 2008; 10 (1): 95-6.

7. Kaneko H, Tomomasa T, Kubota Y, Todokoro M, Kato M, Miyazawa R, Suzuki T, Hatori Y, Kunimoto F, Yamamoto K, Morikawa A. Pharmacobezoar complicating treatment with sodium alginate. J Gastroenterol 2004; 39 (1): 69-71.

8. Bogacz K, Caldron P. Enteric-Coated Aspirin Bezoar: Elevation of Serum Salicylate Level by Barium Study. Am J Med 1987; 83: 783-6.

9. Buckley N, Dawson AH, Howarth D, Whyte IM. Slow-release verapamil poisoning. Use of polyethylene glycol whole-bowel lavage and high-dose calcium. Med J Aust 1993; 158 (3): 2024.

10. Soporer KA, Manning JJ. Massive ingestion of sustained release verapamil with a concretion and bowel infarction. Ann Emerg Med 1993; 22 (3): 603-5.

11. Reid T, Rubins JB, Levine J, DeCelles J, Silvis, S. Colonic medication bezoar from extended-release nifedipine and procainamide. Arch Fam Med 1995; 4 (8): 715-7.

12. Niezabitowski LM, Nguyen BN, Gums JG. Extended-Release Nifedipine Bezoar Identified One Year After Discontinuation. Ann Pharmacother 2000; 34: 862-4.

13. Bernstein G, Jehle D, Bernaski E, Braen GR. Failure of gastric emptying and charcoal administration in fatal sustained-release theophylline overdose: pharmacobezoar formation. Ann Emerg Med 1992; 21 (11): 1388-90.
14. Hutter D, Akgun S, Ramamoorthy R, Dever LL. Medication bezoar and esophagitis in a patient with HIV infection receiving combination antiretroviral therapy. Am J Med 2000; 108 (1): 684-5.

15. Amidon GL, Dressman JB. Enteric Coated Aspirin circumventing Gastric Retention. J Rheumatol 1985; 12 (2): 387-8.

16. Ray MJ, Padin R, Condie JD, Halls JM. Charcoal Bezoar: Small-Bowel Obstruction Secondary to Amitryptyline Overdose Therapy. Dig Dis Sci 1988; 33 (1): 106-7.

17. Dedes KJ, Schiesser M, Scha “fer M, Clavien PA. Postoperative Bezoar Ileus After Early Enteral Feeding. J Gastrointest Surg 2006; 10 (1): 123-7.

18. Scherer A, Corrêa FAR, Corrêa ER, Corrêa ECR. Doença Diverticular do Cólon. Complicação por estenose com formação de bezoar. Relato de um caso. Rev bras Coloproct 1985; 5 (3): 140-2.

19. Ramos R, Herrera M, Hervias D, Duarte P, Vicente C, Casteleiro C. Oclusão intestinal por bezoar resolvida com CocaCola®. J Port Gastrenterol 2007; 14: 153-4.

20. Wang YG, Seitz U, Li ZL, Soehendra N, Qiao XA. Endoscopic management of huge bezoars. Endoscopy 1998; 30 (4): 371-4.

21. Forbes A, Cartwright A, Marchant S, McIntyre P, Newton M. Review article: oral, modified-release mesalazine formulations - proprietary versus generic. Aliment Pharmacol Ther 2003; 17 (10): 1207-14.

22. Layer PH, Goebell H, Keller J, Dignass A, Klotz U†. Delivery and Fate of Oral Mesalamine Microgranules Within the Human Small Intestine. Gastroenterology 1995; 108: 1427-1433.

23. Tuðcu-Demiröz F, Acartürk F, Takka S, Konup-Boyunaða Ö. Evaluation of alginate based mesalazine tablets for intestinal drug delivery. Eur J Pharm Biopharm 2007; 67: 491-497.

24. Dignass A, Marteau P. Mesalamine in the Treatment of Active Crohn's Disease. Gastroenterology 2005; 128 (1): 245-6.

\section{Endereço para correspondência:}

ADRIANA SANTOS NEVES TRECE

Rua Barata Ribeiro 96 / 1212 - Copacabana

Rio de Janeiro - RJ

CEP: 22011-002

Tel: 21 3064-2366

E-mail: rafatrece@gmail.com 